\title{
Control of the convective dynamo in close binaries by tidal locking
}

\section{Is V471 Tauri the template?}

\author{
L. Rottler ${ }^{1, \star}$, C. Batalha ${ }^{2}$, A. Young ${ }^{3}$, and S. Vogt ${ }^{4}$ \\ ${ }^{1}$ Institute for Geophysics and Planetary Physics, University of California, Santa Cruz, Earth and Marine Sciences Building, \\ 1156 High Street, Santa Cruz, CA 95064, USA \\ 2 Observatório Nacional, Coordenação de Astronomia e Astrofísica, Rio de Janeiro RJ 20921-400, Brasil \\ e-mail: celso@on.br \\ 3 Astronomy Department, San Diego State University, 5500 Campanile Drive, San Diego, CA 92182, USA \\ e-mail: young@mintaka.sdsu.edu \\ ${ }^{4}$ Board of Studies in Astronomy and Astrophysics, University of California, Santa Cruz, Santa Cruz, CA 95064, USA \\ e-mail: vogt@ucolick.org
}

Received 15 April 2002 / Accepted 11 June 2002

\begin{abstract}
We report additional observations of the phase dependent behavior of the chromospheric $\mathrm{H} \alpha$ feature associated with the K-dwarf component of V471 Tau. Previously, all such observations have shown a sharply defined maximum of emission near the middle of the transit phase of the white dwarf across the disk of the K-dwarf. The precision and the reproducibility of that phase lock led many previous observers to conclude that the observed emission was due to chromospheric re-processing of ultraviolet radiation incident upon the K-dwarf from the white dwarf. Comparing these observations with previously published observations, we show that the emission strength has been steadily diminishing between epoch 1985 and epoch 1992 whence it has essentially vanished. The ultraviolet flux from the white dwarf is constant so the resulting re-processed chromospheric emission cannot be time dependent. This leads to the conclusion that most of the emission has been due to magnetically active regions located preferentially at the sub-stellar point of the white dwarf. We propose that tidal coupling in this close binary system preferentially affects the transfer of magnetic energy, coupling the internal dynamo of the K-dwarf to the outer envelope, giving a preferred longitude to magnetic activity. We further suggest that this mechanism may be common in most short period binary systems and in many cases may be the dominant mechanism causing the observed $\mathrm{H} \alpha$ behavior.
\end{abstract}

Key words. stars: activity - stars: binaries: eclipsing - stars: chromospheres - stars: individual: V471 Tau - stars: magnetic field - stars: white dwarfs

\section{Introduction}

Over the three decades since its discovery, V471 Tau has rewarded its many observers with new discoveries of astrophysical phenomena, and is apparently continuing in that fine tradition. The strong, complex, and variable feature at $\mathrm{H} \alpha$ has been a focus of much of the observational spectroscopy of this system. Many observers of the $\mathrm{H} \alpha$ line have reported that the feature goes into emission when the hemisphere of the $\mathrm{K}$-dwarf facing the white dwarf is visible to us, and reverts back to an absorption feature when the opposite hemisphere becomes visible during the eclipse of the white dwarf. (Young et al. 1988; Young et al. 1991 (YRS91); Skillman \& Patterson 1988.) Similar behavior observed in other chromospheric

Send offprint requests to: L. Rottler,

e-mail: rottler@es.ucsc.edu

* Formerly with UCO Lick Observatory. diagnostics, such as the $\mathrm{Ca}$ II $\mathrm{H}$ and $\mathrm{K}$, and the $\mathrm{Ca}$ II infrared triplet provides evidence that the activity is coming from the chromosphere of the K-dwarf. However, the $\mathrm{H} \alpha$ absorption profile, when compared with similar profiles from inactive K-dwarfs has a much smaller equivalent width, suggesting that it is partially filled by chromospheric emission. Given that there was evidence for magnetically induced activity all around the K-dwarf (Young et al. 1983), there was no reason to think that it was particularly concentrated in some restricted zone of longitudes near the sub-stellar point. The greatly enhanced $\mathrm{H} \alpha$ emission observed to be concentrated at that location was, therefore, interpreted as originating from re-processing of incident ultraviolet radiation from the white dwarf (Young et al. 1988; Skillman \& Patterson 1988). Given the evidence available over those years it was a plausible scenario; perhaps even the most likely one. 
The additional observations reported in this paper cast some doubt upon that interpretation. The incident radiation from the white dwarf is expected to be invariant in both its magnitude and its geometry. Hence we would not expect to observe such a secular decrease in the intensity of the re-processed $\mathrm{H} \alpha$ emission. The intensity of $\mathrm{H} \alpha$ emission in the vicinity of the sub-stellar point of the white dwarf suggests that the primary source of the emission is localized magnetic activity. Such activity is known to be cyclical in chromospherically active latetype stars (Hall 1990). The activity cycle has a period on the order of 6 to 7 years for the K-dwarf in V471 Tau (Ibanoglu et al. 1994).

The observations presented here include those made at the Kitt Peak National Observatory in November 1990, and those made at Lick Observatory in October and November 1992. The observations made at the Kitt Peak National Observatory used the same instrument configuration reported by YRS91, with an effective resolution of $0.44 \AA$ and a typical signal-to-noise ratio of 200. The Kitt Peak spectra were reduced with standard spectral analysis routines developed by G. Woods at HAO; later upgraded and ported to UNIX by one of the authors, L. Rottler in 1987 at Photon Research Associates in San Diego.

The observations made at the Lick Observatory used the $3 \mathrm{~m}$ telescope with the Hamilton Echelle Spectrograph (Vogt 1987). The detector used was an LN2 cooled TI $2054 \times 2054$ $\mathrm{CCD}$ at the Coude focus. For these observations the effective resolution was $0.14 \AA$ at $\mathrm{H} \alpha$ with a typical signal-to-noise ratio of 100 to 150 . The size of the chip covered a large part of the echelle format allowing simultaneous measurement of the chromospheric lines of interest: from the $\mathrm{Ca}$ II $\mathrm{H} \& \mathrm{~K}$ in the blue to the Ca II infrared triplet at $8662 \AA$. Typical exposures were $20 \mathrm{~min}$ long and the average seeing was 0.5 arcsec which combined to give the quoted signal-to-noise ratio. The data were reduced in a standard way with the IDL based routines developed by Valenti (1994).

Radial velocity corrections were then performed to convert all the observations into the rest frame of the K-dwarf. The system configuration at each particular phase was then computed using the ephemeris data taken from Skillman \& Patterson (1988) and the projected velocity of the K-dwarf companion derived assuming a nearly edge on inclination for the plane of the orbit (Young \& Nelson 1972). For purposes of comparison we include some observations from 1985 published by YRS91.

\section{Results}

Figure 1 shows a superposition of the $\mathrm{H} \alpha$ profile from the 1985 observations and from the 1992 observations, normalized to the same scale, at the transit phase $(\phi=0.5)$ of the white dwarf across the disk of the K-dwarf. The significant reduction of the emission strength over the separation of seven years is readily apparent.

Figure 2 displays the $\mathrm{H} \alpha$ profile in order of orbital phase for the three nights of observation at Lick Observatory. Superimposed upon those plots is the $\mathrm{H} \alpha$ profile of an inactive $\mathrm{K}$-dwarf used as a reference source. The dichotomy of emission at the transit phase $\phi=0.5$ and absorption at the occultation phase $p h i=0.0$ is still apparent from these data, but very much

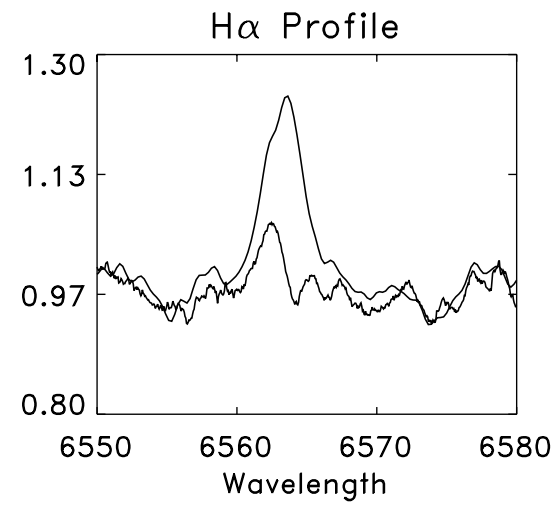

Fig. 1. $\mathrm{H} \alpha$ emission line observed at different epochs at the phase $\phi=0.5$ (the white dwarf transit). The 1985 data indicates chromospheric emissivity larger than that of the 1992 observations.

reduced from previous epochs. The variations of the profile and of its asymmetry are consistent with reports by other observers at earlier epochs. However, we call attention to the sudden appearance of strong $\mathrm{H} \alpha$ emission at phase $\phi=0.851$ on the night of 10 Nov. 1992, suggesting that a significant hemispheric flare erupted and subsided within a short timescale.

Figures $3 \mathrm{a}-\mathrm{c}$ display the measured values of the equivalent width of $\mathrm{H} \alpha$ as a function of phase for the three epochs (1985, 1990, and 1992). These equivalent widths are measured differentially from the quiescent reference star, so they can be taken to be the amount of excess emission contributed by the chromosphere of the K-dwarf companion of V471 Tau. From that perspective, the equivalent width is always positive (emission) even during the occultation phases when the observed profile appears to be in absorption. Significant flare events that could be verified are noted on each of the plots. We call attention to the systematic decrease of the sharply phase-dependent emission that occurs during the transit phases.

The excess of line emission as a function of phase is not exclusive to the $\mathrm{H} \alpha$ line. The $\mathrm{Ca}$ II infrared triplet lines also show similar behavior, although in a less dramatic way (Fig. 4). The eruption of a flare during the phase $\phi=0.85$ of November 10 th is immediately evident in the infrared triplet.

\section{Discussion}

In view of our analysis of the epoch 1992 data, if re-processing of the ultraviolet radiation incident on the K-dwarf represents any portion of the observed $\mathrm{H} \alpha$ emission at the sub-stellar point, it must be a relatively small contribution. Previous estimates of the viability of the UV re-processing mechanism were largely based on the assumption that the chromosphere was optically thin to the Balmer lines (Skillman \& Patterson 1988). The match between the total chromosphere line emission and the incident UV radiation provided circumstantial support for the radiative mechanism. However, consistent calculations indicate that these lines are optically thick for typical active chromospheres (Thatcher et al. 1991).

In addition to the main $\mathrm{H} \alpha$ chromospheric emission around $\phi=0.5$, evidence of an external hot plasma emission region at a radial velocity between that of the first Lagrangian point and 


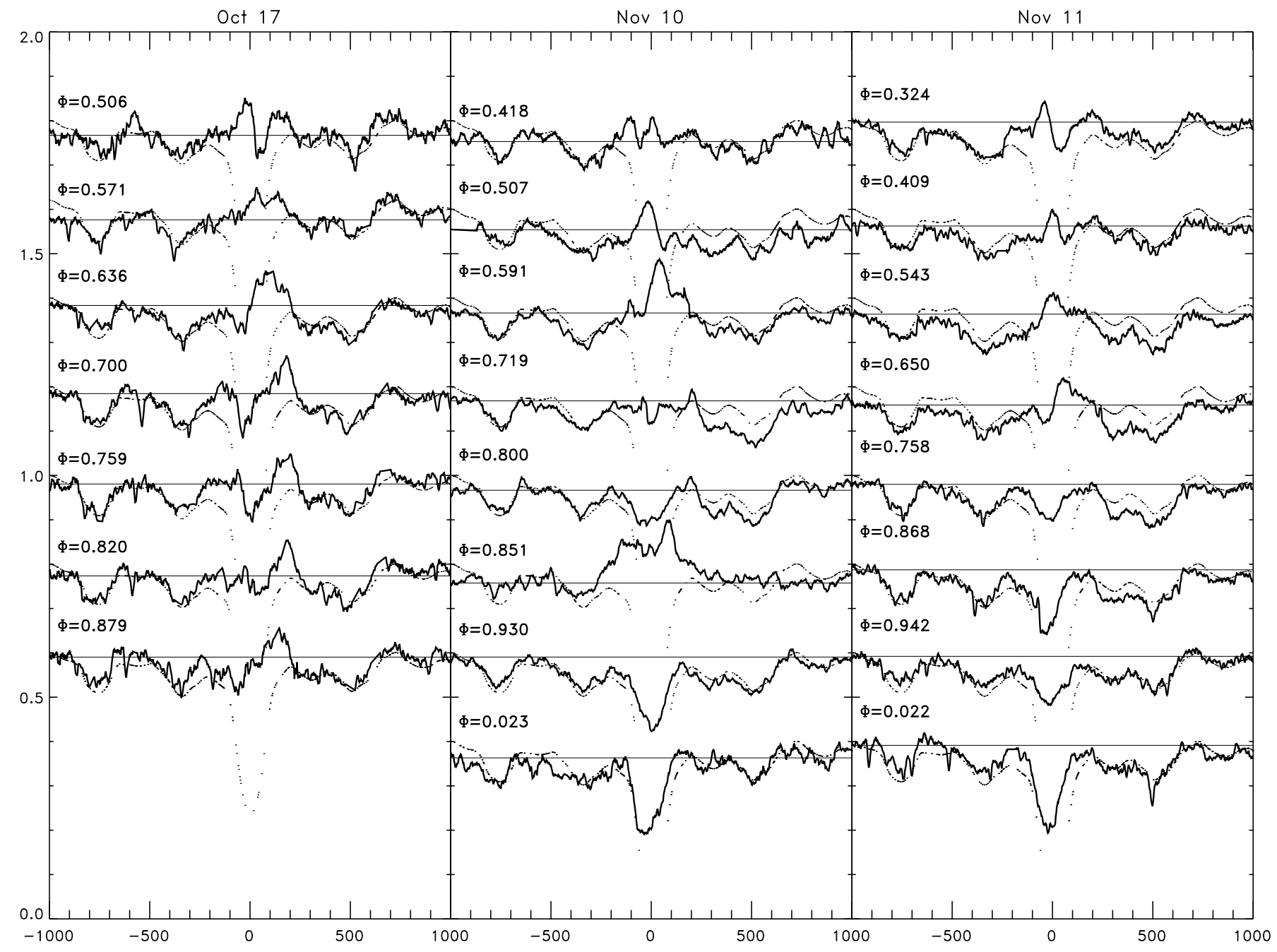

Fig. 2. H $\alpha$ profiles for the 1992 observations. Each panel shows the K-dwarf profile corrected to the stellar rest frame, together with that of an inactive template (dotted line). A plasma emission external to the stellar atmosphere can be seen in the October 17th observations.

that of the white dwarf was shown by YRS91. Those authors also noted that stochastically independent ejections of plasma consistently reproduced the same dynamical behavior with respect to the observed radial velocity of the external plasma. Similarly, the $\mathrm{H} \alpha$ time series shown in Fig. 2 indicates the presence of large structures of plasma in emission in the 1992 data in spite of the overall decrease in the chromospheric Balmer emission. The major plasma emission at $150 \mathrm{~km} \mathrm{~s}^{-1}$ seen at $\phi=0.571$ (October 17) migrates to a maximum emission of $200 \mathrm{~km} \mathrm{~s}^{-1}$ at $\phi=0.759$, near quadrature, and recedes back to line center at $\phi=0.879$, basically confirming the results referred to in YRS91, and extending the phase coverage to almost $\phi=0.9$. Since the projected rotation of the K-dwarf is $90 \mathrm{~km} \mathrm{~s}^{-1}$, the emission is likely to arise in the region between both stars.

UV re-processing cannot explain the emission from these large structures lying above the atmosphere. Again, we argue that the presence of this bright $\mathrm{H} \alpha$ emitting region is suggestive of the presence of plasma, trapped by magnetic field lines that are anchored on the surface of the K-dwarf located close to the sub-stellar point of the white dwarf. Damping of Alfven waves might play a major role in providing the necessary heating mechanism to the gas. Large magnetic flux tubes would provide the structure required to transport the plasma from flare activity at the surface of the K-dwarf to the regions above the surface where the emission is observed. Large magnetic structures can be supported at such large distances from the star, as discussed by Ferreira \& Jardine (1995). They show that magnetic filaments or loops can be stable out to 3 to 9 times the stellar radius. It was suggested by Lim et al. (1996), that on V471 Tau large magnetic flux loops interacting with the magnetosphere of the white dwarf could give rise to the radio emission observed from plasma trapped in this region. This could explain the consistency in the observed radial velocity of the emitting region. Their model of large coronal loops interacting with the white dwarf magnetosphere is not inconsistent with IUE observations (Guinan et al. 1986) nor the optical emission from external plasma reported by YRS91.

One could argue that the observed secular decrease in $\mathrm{H} \alpha$ emission is explained by a decreasing accretion flow from the 


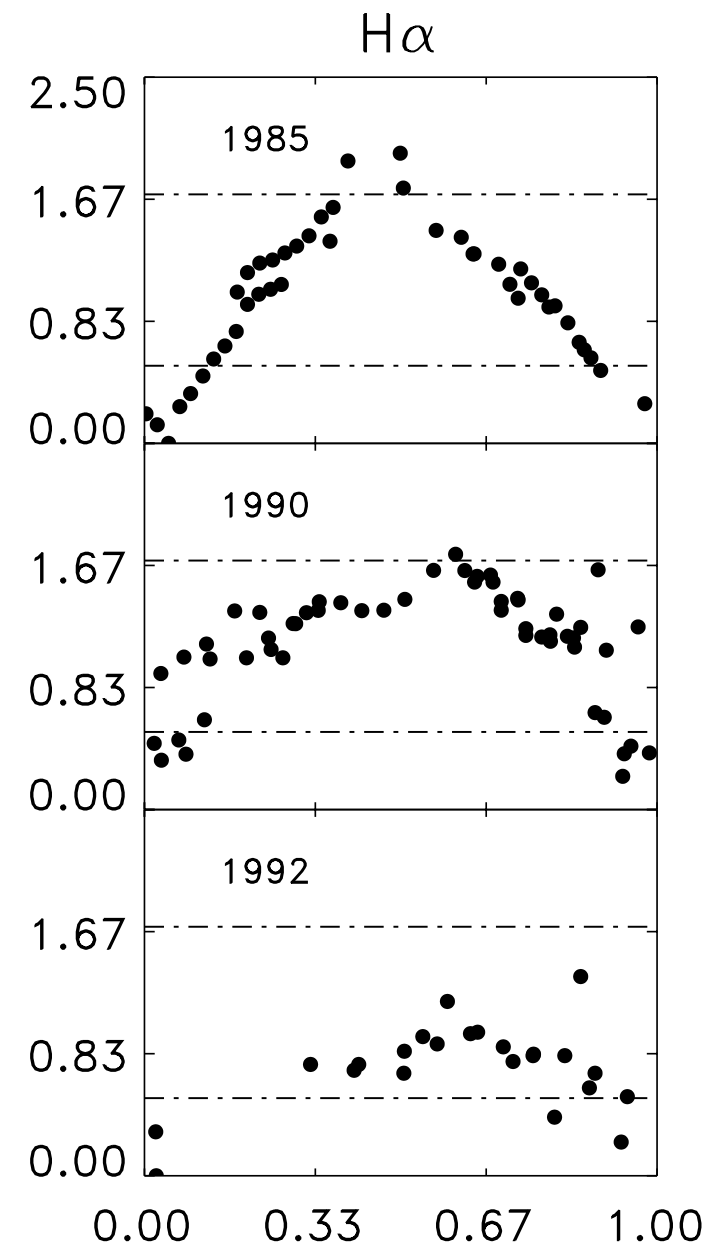

Fig. 3. The secular decrease of the global chromospheric emission of V471 Tau. The horizontal dotted lines represent the average emission of the low and ultra fast K-dwarf rotators of the Pleiades (Soderblom et al. 1993). a) The top panel shows the $\mathrm{H} \alpha$ equivalent width at maximum strength. b) The middle panel shows chromospheric activity at a lower, intermediate, strength in 1990. c) The bottom panel shows $\mathrm{H} \alpha$ activity at minimum strength in 1992 .

first Lagrangian point. However it is known that the K-dwarf does not overflow its Roche lobe and continuous mass transfer due to winds or other mechanisms has not been detected at a measurable level (Bruhweiler \& Sion 1986; Robinson et al. 1988).

Our data indicate that most of the hemispheric emission observed on the inner hemisphere of the K-dwarf is the result of a convergence of magnetically active regions around the substellar point of the white dwarf. In fact, as shown in Fig. 3, there is not only an increase in brightness of emission of $\mathrm{H} \alpha$ on the hemisphere facing the white dwarf but also a dramatic decrease in brightness on the opposite hemisphere, $(\phi=0.0)$. The excess of emission measured when the white dwarf was transiting the K-dwarf companion $(\mathrm{H} \alpha>2.0$ at $\phi=0.5$ in 1985) was substantially larger than that seen during the eclipsing phase of the white dwarf $(\mathrm{H} \alpha<0.3)$. Granting we are witnessing a whole range of active stages that are phase dependent, it is worthwhile to compare these measures with those of a sample of active stars.

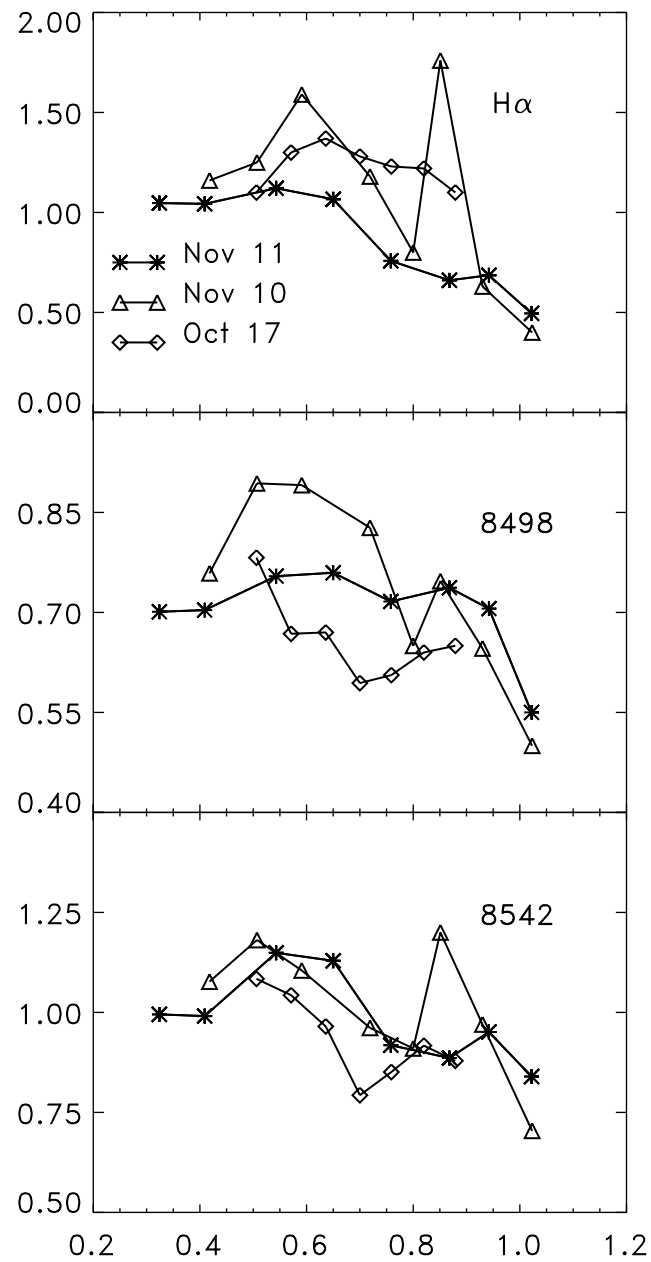

Fig. 4. The 1992 observations include the CaII infrared triplet lines. The equivalent width of the residual profile is displayed.

Soderblom et al. (1993) display the $\mathrm{H} \alpha$ and Ca II infrared triplet emission for a large sample of Pleiades single stars. Their method of measuring the equivalent widths is similar to that adopted in this work, allowing direct comparison of the line measures. Figure 3 indicates the average $\mathrm{H} \alpha$ for the slow rotators and the fast rotators $\left(v \sin i>20 \mathrm{~km} \mathrm{~s}^{-1}\right)$ of the Pleiades (dotted lines). Based upon the Pleiades observations, the strength of the chromospheric emission detected during the eclipse of the white dwarf is significantly less than that for rapidly rotating single stars. Since V471 Tau is a fast rotator this observation may be evidence for the concentration of active chromospheric regions at the sub-stellar point of the white dwarf; i.e., a longitudinal distribution of magnetically induced activity that is not observed on rapidly rotating single stars.

If magnetically active regions can reach the surface of a star at any and all longitudes, it seems curious that on the Kdwarf component of V471 Tau they prefer a special longitude, aligned with the white dwarf companion. Since our observations rule out radiative effects from the white dwarf as the cause of this alignment, all that remains to make that longitude special is the strong tidal coupling of the white dwarf to the K-dwarf. Photometric evidence of spot modulation leaves no doubt that the K-dwarf is rotating synchronously with the 
orbit (Ramseyer \& Hatzes 1995). Taken collectively, the evidence suggests that the tidal distortion of the K-dwarf star has at least affected the transfer of magnetic energy from the internal stellar dynamo such as to make it easier for magnetic flux to reach the surface of the star in the region where the gradient in the gravitational potential is least. The resultant effect is to concentrate magnetic activity at the sub-stellar point of the WD.

In the case of V471 Tau the WD is only 3.85 stellar radii away from the center of the K-dwarf star. Hence the inner hemisphere of the K-dwarf is only 2.85 stellar radii away, while the outer hemisphere is 4.85 stellar radii away from the WD. Since the ratio of the differential tidal force follows an inversecubed distance law, the ratio of the forces due to the WD on the two hemispheres of the K-dwarf is $(2.85 / 4.85)^{3}$ or 0.20 . In the more familiar system of the Earth and its moon, the separation of the two bodies is about 60 times the radius of the Earth, leading to a hemispheric ratio of 0.90 for the differential tidal forces. Thus, while the two hemispheric tides on the Earth are nearly symmetrical, those on the K-dwarf are substantially asymmetrical.

We propose that the dominant mechanism for the observed $\mathrm{H} \alpha$ emission around $\phi=0.5$ is the distortion of the convective dynamo of the K-dwarf star by tidal interaction with its white dwarf companion. This favors the emergence of active regions concentrated at the sub-stellar point on the inner hemisphere of the K-dwarf giving rise to the observed $\mathrm{H} \alpha$ emission at this preferred longitude. This being the case one should expect to see this effect in other tidally locked, short period, binaries that are chromospherically active. The telltale signature for this effect is an increase in intensity of $\mathrm{H} \alpha$ emission around phase $(\phi=0.5)$ and a secular variation of this emission correlated with the magnetic activity cycle of the star.

Several V471 Tau analogs have been found that exhibit the $\mathrm{H} \alpha$ emission feature at $\phi=0.5$. A significant sample of these whose system parameters are known or can be calculated are listed in Table 1. Many of these V471 Tau analogs are systems consisting of a hot DA white dwarf and an M-dwarf. In each of the tabulated systems the hot companions have temperatures ranging from $30000 \mathrm{~K}$ to $60000 \mathrm{~K}$, and periods ranging from $1 / 3$ to 9 times that of V471 Tau. It is only in the systems with a close, very hot DA WD companion that one would expect UV re-processing to be a significant effect.

Based upon our observations and findings we have taken V471 Tau to be a benchmark for radiative re-processing of ionizing UV radiation incident upon the hemisphere of a cool star from its hot companion. Using the grid of model atmospheres for hot white dwarfs tabulated by Wesemael (1980) we integrated the emergent flux over all tabulated wavelengths shorter than $912 \AA$. From the published orbit solutions and other physical parameters given by those authors, we calculated the geometrical dilution factor for the radiation field of the WD that is incident upon the cool companion. Combining those calculations permits us to estimate the flux of ionizing radiation impinging upon each of the cool companion stars, and those values are tabulated in Table 1.

We then normalized the incident flux to that for the K-dwarf of V471 Tau. Our study suggests that systems in which the
Table 1. Ionizing flux in analog systems normalized to V471 Tau ${ }^{a}$.

\begin{tabular}{llll}
\hline \hline System & UV Flux & Norm Flux & Sources $^{b}$ \\
\hline Feige 24 & 9.7 E09 & 3 & $1,10,11,12$ \\
NN Ser & 1.3 E11 & 40 & $2,10,11$ \\
PG1224 + 309 & 1.4 E09 & 0.4 & $3,10,11$ \\
EUVE J0720 + 317 & 2.3 E10 & 7 & 4 \\
EUVE J1016-053 & $1.5 \mathrm{E} 10$ & 5 & 4 \\
EUVE J2013 + 400 & $2.3 \mathrm{E} 10$ & 7 & 4,10 \\
CASE 1 & 5.9 E05 & $1.8 \mathrm{E}-04$ & 5,12 \\
HZ 9 & $4.4 \mathrm{E05}$ & $1.3 \mathrm{E}-04$ & $6,10,11,12$ \\
GD 448 & 4.6 E05 & $1.4 \mathrm{E}-04$ & $7,10,11$ \\
V471 Tau & $3.3 \mathrm{E09}$ & 1.0 & $8,9,11,12$ \\
\hline
\end{tabular}

a Column 1 lists the name of the system, Col. 2 tabulates the ionizing flux at the surface of the cool star from the WD in units of erg $/ \mathrm{s} / \mathrm{cm}^{2} / \mathrm{ster}$, Col. 3 tabulates the ionizing flux normalized to V471 Tau, and Col. 4 lists the references.

${ }^{b} 1$ - Vennes et al. (1994), 2 - Woods et al. (1991), 3 - Orosz et al. (1999), 4 - Vennes et al. (1999), 5 - Lanning (1982), 6 - Stauffer (1987), 7 - Maxted et al. (1998), 8 - Young et al. (1972), 9 - Skillman et al. (1988), 10 - Marsh (2000), 11 - Hillwig et al. (2000), $12-$ Ritter (1986).

normalized flux is less than or comparable to unity cannot drive $\mathrm{H}$-alpha into emission by re-processing UV radiation from the hot companion. Systems with values for the normalized flux that is larger than unity cannot be ruled out as candidates to drive $\mathrm{H}$-alpha into emission by re-processing. However, we note that nearly all such systems have values for the normalized flux that is of the same order of magnitude as that for V471 Tau, so it seems unlikely that they are radiatively driven. Only the one system, NN Ser, with a normalized flux ratio of 40 , is a candidate for radiative re-processing.

The geometric dilution factors of all these binaries are similar and small (averaging 0.004), so it is apparent that the deciding factor for radiative re-processing is the temperature of the hot companion. That is not surprising in view of the fact that the emergent radiation from such hot sources is not unlike that of a blackbody, and the peak intensity from a blackbody is proportional to the fifth power of its temperature. Since the integrated ionizing flux is in a narrow window close to the peak intensity, the proportionality is closer to the fifth power than to the fourth power of the temperature. The latter being the result of integrating the flux over all wavelengths.

\section{Summary}

In light of the additional spectrographic data taken in 1990 and 1992 combined with those taken in 1985 there is clear evidence of a marked secular decrease in $\mathrm{H} \alpha$ emission at $\phi=0.5$. We argue that re-processing of UV radiation from the white dwarf can no longer be invoked as the main mechanism to explain the observed chromospheric emission from the inner hemisphere of the K-dwarf. If it were, the $\mathrm{H} \alpha$ emission would be expected to remain constant over time, given the constancy of the UV flux from the white dwarf. In fact the intensity of the $\mathrm{H} \alpha$ emission appears to be consistent with the period of mean 
luminosity of the star, measured by Ibanoglu et al. (1994), to be around 6 to 7 years, indicating a solar-like cycle of magnetic activity. We have argued therefore, that the $\mathrm{H} \alpha$ emission at $\phi=0.5$ is primarily due to magnetic heating from the asymmetric tidal distortion of the convective dynamo of the Kdwarf star by its WD companion. We further suggest, based upon available evidence, that this may be a general mechanism in very short period detached binary systems. The notion that strong tidal coupling alters internal dynamos in rotating convective stars may have considerable significance in the attempt to produce a proper theory for such dynamo action.

As it has in the past the V471 Tau system has proven itself to be a wonderful astrophysical laboratory for the study of short period binary systems and has once again rewarded us with new insights that have shed more light on our understanding of binary interactions.

Acknowledgements. The authors would like to thank Mount Laguna Observatory (MLO), Paul Etzel in particular, for providing the facilities and support to complete this work. In addition one of the authors, $\mathrm{CB}$, is grateful to the Board of Studies in Astronomy and Astrophysics for computer support while visiting UCSC. We would also like to thank the reviewer, C. Ibanoglu, for his timely review and helpful comments.

\section{References}

Bruhweiler, F. C., \& Sion, E. M. 1986, ApJ, 304, L21

Ferreira, J. M. T. S., \& Jardine, M. 1995, A\&A, 298, 172

Guinan, E. F., Wacker, S. W., Baliunas, S. L., Loeser, J. G., \& Raymond, J. C. 1986, in New Insights in Astrophysics: Eight Years of UV Astronomy with IUE, ESA SP-263, 197

Hall, D. S. 1990, in Active Close Binaries, ed. C. Ibanoglu (Dordrecht: KLuwer), 95
Hillwig, T. C., Honeycutt, R. K., \& Robertson, J. W. 2000, AJ, 120, 1113

Hrivnak, B. J., Guinan, E. F., \& Lu, W. 1996, ApJ, 455, 300

Ibanoglu, C., Keskin, V., Akan, M. C., Evren, S., \& Tunca, Z. 1994, A\&A, 281, 811

Lanning, H. 1982, ApJ, 253, 752

Lim, J., White, S. M., \& Cully, S. L. 1996, ApJ, 461, 1009

Marsh, T. R. 1996, New Astron. Rev., 44, 119

Maxted, P. F. L., Marsh, T. R., Moran, C., Dhillon, V. S., \& Hilditch, R. W. 1998, MNRAS, 300, 1225

Orosz, J. A., Wade, R. A., \& Harlow, J. J. B. 1997, AJ, 114, 317

Orosz, J. A., Wade, R. A., Harlow, J. J. B., Thorstensen, J. R., Taylor, C. J., \& Eracleous, M. 1999, AJ, 117, 1598

Ramseyer, T. F., \& Hatzes, A. P. 1995, AJ, 113, 1364

Ritter, H. 1986, A\&A, 169, 139

Robinson, E. L., Clemens, C., \& Hine, B. P. 1988, ApJ, 331, L29

Skillman, D. R., \& Patterson, J. 1988, AJ, 96, 976

Soderblom, D. R., Stauffer, J. R., Hudon, J. D., \& Jones, B. F. 1993, ApJS, 85, 315

Stauffer, J. R. 1987, AJ, 94, 996

Thatcher, J. D., Robinson, R. D., \& Rees, D. E. 1991, MNRAS, 250, 14

Vennes, S., \& Thorstensen, J. R. 1994, AJ, 108, 1881

Vennes, S., Thorstensen, J. R., \& Polomski, E. F. 1999, ApJ, 523, 386

Wesemael, F., Auer, L. H., Van Horn, H. M., \& Savedoff, M. P. 1980, ApJS, 43, 159

Wood, J. H., \& Marsh, T. R. 1991, ApJ, 381, 551

Young, A., Klimke, A., Africano, J. L., et al. 1983, ApJ, 267, 655

Young, A., \& Nelson, B. 1972, ApJ, 173, 653

Young, A., Skumanich, A., \& Paylor, V. 1988, ApJ, 334, 397

Young, A., Rottler, L., \& Skumanich, A. 1991, ApJ, 378, L25

Valenti, J. A. 1994, Ph.D. Thesis, University of California, Berkeley

Vogt, S. 1987, PASP, 99, 214 